\title{
La enfermera transcultural y el desarrollo de la competencia cultural
} \section{The transcultural nurse and the development of cultural competence Enfermeira transcultural eo desenvolvimento de competência cultural}

\author{
Elizabeth Castrillón Chamadoira
}

Graduada en Enfermería por la Universidad de Oviedo.

Cómo citar este artículo en edición digital: Castrillón Chamadoira, E. (2015). La enfermera transcultural y el desarrollo de la competencia cultural. Cultura de los Cuidados (Edición digital), 19, 42.

Disponible en: http://dx.doi.org/10.14198/cuid.2015.42.11>

Correspondencia: C/Roncal 20 7H 33208 Gijón (Asturias).

Correo electrónico: elizabeth.castri@gmail.com

Recibido: 16/01//2015; Aceptado: 11/06/2015

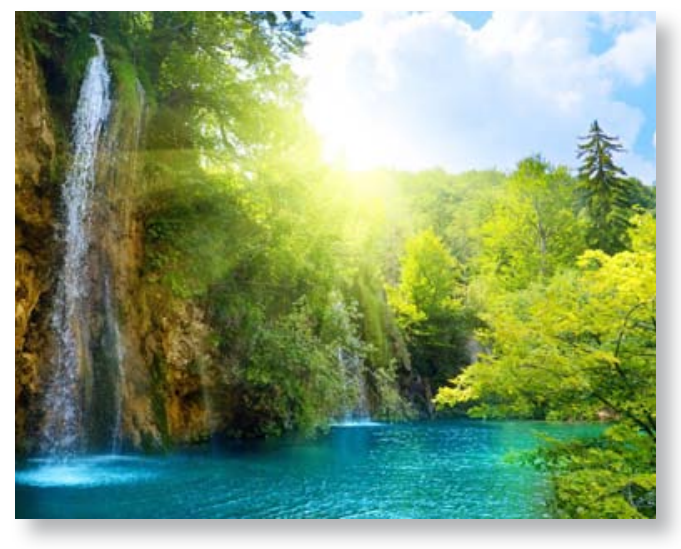

ABSTRACT

Given the increased migration and the need for nurses to adapt to this reality, the purpose of this article is to know how to address the transcultural nursing care.

The method used was based on a literature review with critical and reflective of the literature of national and international reading.

The concept of cultural competence is developed and the models and theories of nursing based on intercultural communication, ethical and legal issues, institutional measures of cultural consideration and incorporating this knowledge in higher education are discussed.

It concludes with the need to acquire cultural competence to facilitate the establish- ment of the therapeutic relationship through understanding and understanding of other cultures, valuing the context of the individual as a transcendental element.

Keywords: transcultural nursing, cultural competency, health communication and emigration and inmigration.

\section{RESUMO}

Dado o aumento da migração e da necessidade de enfermeiros para se adaptar a essa realidade, o objetivo deste artigo é saber como lidar com o cuidado de enfermagem transcultural.

O método utilizado foi baseado em uma revisão da literatura com crítica e reflexiva da literatura de leitura nacional e internacional.

O conceito de competência cultural é desenvolvido e os modelos e teorias de enfermagem com base na comunicação intercultural, questões éticas e legais, medidas institucionais de reflexão cultural e incorporar esse conhecimento no ensino superior são discutidas.

Conclui-se com a necessidade de adquirir competência cultural para facilitar o estabelecimento da relação terapêutica através da compreensão e compreensão de outras culturas, valorizando o contexto do indivíduo como um 
elemento transcendental.

Palavras-chave: enfermagem transcultural, competência cultural, comunicação em saúde e Migração Internacional.

\section{RESUMEN}

Teniendo en cuenta el aumento de las migraciones y la necesidad de las enfermeras de adaptarse a esta realidad, el objetivo de este artículo es conocer cómo debe abordar la enfermera el cuidado transcultural.

El método empleado se basó en una revisión bibliográfica con lectura crítica y reflexiva de la literatura de ámbito nacional e internacional.

Se desarrolla el concepto de competencia cultural y se analizan los modelos y teorías de enfermería, la base de la comunicación intercultural, los aspectos éticos y legales, las medidas institucionales de consideración cultural y la incorporación de este conocimiento en la enseñanza universitaria.

Se concluye con la necesidad de adquirir competencia cultural para facilitar el establecimiento de la relación terapéutica a través del entendimiento y la comprensión de otras culturas, valorando el contexto de la persona como un elemento trascendental.

Palabras clave: enfermería transcultural, competencia cultural, comunicación en salud y migración internacional.

\section{INTRODUCCIÓN}

El concepto de cuidados como base para la enfermera y el de cultura proveniente de la antropología, se entrelazan y cobran gran importancia para la labor asistencial de la profesión. Esta unión abarca un concepto más novedoso y amplio, introducido inicialmente por Madeleine Leininger en los años 50, conocido como Enfermería Transcultural, enunciado en su "Teoría de la diversidad y universalidad de los cuidados".

Como enfermeras debemos ser conscientes que vivimos en una realidad multicultural, que propicia determinadas situaciones sanitarias a las que debemos saber dar respuesta como responsables del cuidado. Ahora no solo nos encontramos en nuestra ciudad de origen con estas situaciones, sino que también, nos podemos ver ejerciendo la profesión en otro país con otro entorno y cultura. Un ejemplo muy cercano es la "masiva emigración de profesionales de la salud en busca de trabajo, de mejoras en las condiciones laborales, o bien para conocer otras experiencias que permitan un mayor enriquecimiento personal y profesional" (Bonill, 2012).

Actualmente vivimos en un mundo globalizado, donde el mayor reto para la sociedad está en adaptarse y cubrir las necesidades que la inmigración plantea en nuestro entorno. Siendo conscientes de esta realidad, es preciso analizar cómo son esos movimientos migratorios. Según datos del Ministerio de Trabajo y Asuntos Sociales, en junio de 2013 había un total de 5.503.977 extranjeros residentes en España, de los cuales, 2.634.199 eran mujeres (Ministerio de Empleo y Seguridad Social, 2013). Vemos por tanto, que en los movimientos migratorios la presencia de mujeres inmigrantes es casi equiparable a la de hombres y esto supone también diferenciar las intervenciones sanitarias, pues es inevitable tener en cuenta que las necesidades de las mujeres cambian con respecto a las de los hombres desde una visión psicológica, social y física, y que es preciso ofertar una asistencia sanitaria que cubra esta demanda, para aportar calidad a nuestro trabajo y al desarrollo de la sociedad.

Por todo lo expuesto, el propósito de este 
trabajo es poner de manifiesto la necesidad de avanzar en éste área de conocimiento de la enfermería transcultural para proporcionar una respuesta de enfermería culturalmente competente, conociendo cómo debe abordar la enfermera el cuidado con personas de otra cultura, destacando la importancia de la comunicación intercultural en el proceso de relación terapéutica, de explorar las opciones que la sociedad puede ofrecer como apoyo a la integración, así como subrayar la importancia de incluir en los programas académicos de las universidades de enfermería formación relativa a los cuidados basados en aspectos culturales.

\section{MÉTODO}

El método ha consistido en realizar una revisión bibliográfica de artículos publicados preferentemente en los últimos cinco a diez años, en las bases de datos: Cuiden, Cinhal, Lilacs, Cuidatge, Scielo y Dialnet, donde pude obtener información acerca de cómo se está abordando el cuidado que las enfermeras proporcionan, teniendo en cuenta las consideraciones culturales. De igual modo, se han analizado otros documentos y publicaciones que hacen referencia a la salud en el perfil de inmigrante.

He incluido todos aquellos artículos que centraban su contenido en la competencia cultural en la práctica y en la formación académica y excluido aquellos que no tuviesen base científica o no respondieran al objetivo del tema de revisión.

\section{DESARROLLO DEL TEMA}

Madeleine Leininger, enfermera pionera en introducir el concepto de cultura en los cuidados, define ésta como "las creencias, valores y modos de vida de un grupo particular, que son aprendidas y compartidas, y por lo gene-

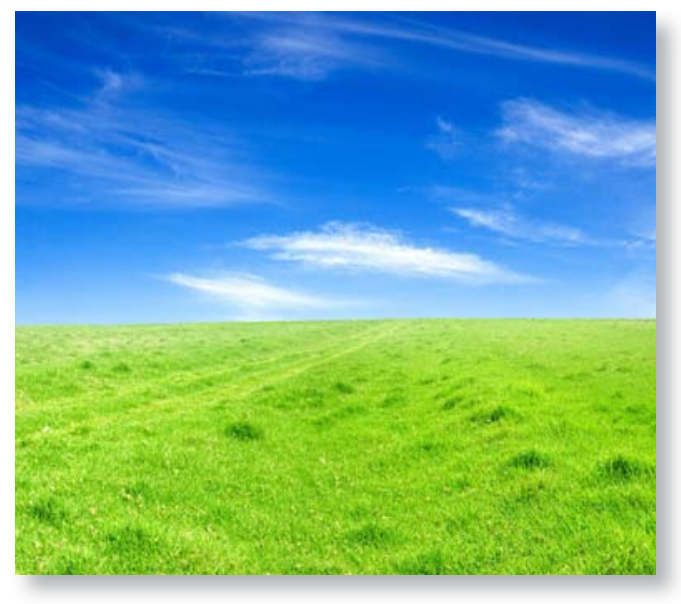

ral transmitidas de forma intergeneracional influenciando las formas de pensamiento $y$ acción" (McFarland, 2007). Otra definición de cultura en el ámbito de la enfermería española viene dada de la mano de J. Siles, que señala que además de basarse en conductas y pensamientos, incluye sentimientos que se implican en el proceso de satisfacción de las necesidades de un grupo humano (Bonill, 2012).

Como enfermeras, debemos preguntarnos de qué manera la cultura puede influir en el cuidado que damos. Una de las fases del proceso de atención de enfermería se basa en valorar al individuo como un ser único e integral, con necesidades biológicas, psicológicas y sociales. Debemos ser conscientes que introducir el ámbito social en la valoración de enfermería es un aspecto significativo a tener en cuenta, ya que nuestras intervenciones deben contemplar por tanto el entorno y la sociedad como determinantes en el proceso de salud de una persona.

Parece lógico pensar que el concepto de cultura que es entendido en enfermería para ofrecer cuidados, sea aquel que pueda dar respuesta a las necesidades de las personas de manera coherente a sus pensamientos y sentimientos, es decir, entender las acciones que las personas toman porque sencillamente es su 
manera de pensar. Sin embargo, resultaría más útil hacer un ejercicio de amplitud, y considerar la cultura en el cuidado, no solo desde las características individuales que puede presentar una persona concreta en relación a la salud y enfermedad, sino adiestrarse en la realidad socio-política y en la participación de la comunidad que influye en esa persona, entrando aquí a formar parte el contexto como elemento fundamental.

Tomar conciencia y aceptación de las diferencias culturales y valores propios es un ejercicio que requiere compromiso de la enfermera, pues supone huir de los estereotipos y superar los prejuicios con el fin de establecer una relación efectiva, desde el respeto de lo que no es propio y con conciencia cultural. En palabras de Plaza del Pino, consiste más en "aprender con el otro que sobre el otro, ver a la persona antes que al extranjero y personalizar los cuidados a las características personales como siempre hacemos" (Plaza del Pino, 2011). Por todo ello, se ve que "si concebimos la cultura como parte importante de la identidad de la persona, ésta debe ser sin duda reconocida en el cuidado de enfermería" (Lipson, 2000).

La Enfermería Transcultural como concepto se basa en una ideología, una manera de enfocar el cuidado hacia la consideración cultural en su práctica. En España, el problema de estas teorías y modelos es que parece ser que no son prácticos ni útiles para su uso por las enfermeras, pues los consideran "discursos amplios y vagos no aplicables a la vida real, $y$ al ser así, las enfermeras no adquieren el compromiso de llevarlos a la acción" (Chamizo, 2005). Es bastante fácil que este problema sea debido a la falta de formación en la universidad con respecto a esta área de conocimiento o los escasos recursos bibliográficos de aplica- ción práctica de estos modelos que existen en nuestro país.

Lo más difícil a mi modo de ver acerca de la teoría de Leininger, es asumir que existe la necesidad de obtener un conocimiento documentado de las culturas para poder usarse como base en la planificación de los cuidados culturales, según los métodos cualitativos de investigación que ella ha desarrollado en su trayectoria profesional, pues esto supone dos esfuerzos añadidos en la rutina diaria de una enfermera asistencial: el primero, estudiar en profundidad al menos las culturas con las que más frecuentemente se va a encontrar en su trabajo y segundo, "romper la barrera gerencial o política que está impuesta en los centros sanitarios en los cuales se producen choques constantes entre culturas, y al no considerar la misma como el elemento clave, provoca que disminuya la calidad en la atención" (Chamizo, 2005).

Leininger afirmó que el objetivo de su teoría no es más que proporcionar cuidados que sean coherentes con la cultura para dar calidad al mismo, y para ello se debe tener conocimiento de la cultura y aplicarlo (McFarland, 2007). Con esta afirmación se puede suponer que todas las enfermeras que trabajen con esta teoría y estudien y analicen las características de cada cultura sabrán proporcionar cuidados culturales. Sin embargo, podríamos caer en el error de considerar determinados aspectos genéricos de la cultura en todas las personas que pertenezcan a un mismo grupo cultural, pues no todos pueden responder de la misma manera ante las situaciones, bien por diversas experiencias de su vida o por la manera de entender el mundo, o incluso por su formación, por lo que quizás sea más práctico y útil entender que proporcionar cuidados culturales debe estar ajustado a la cultura en la forma en 
que cada persona concreta la entiende, la vive y la manifiesta, sin considerar a priori ningún patrón prestablecido que pueda conducir a errores y por lo tanto a fracaso de la relación enfermera - usuario. Creo que la frase que lo definiría es que no atendemos únicamente culturas, sino personas.

Por todo ello, es importante hacer de las teorías y modelos un recurso práctico para las enfermeras, útil en su manejo y sobre todo adaptado a la realidad con la que trabajan. Debemos tener conocimientos de las culturas con las que vamos a trabajar, pero no debemos pensar que es el único instrumento que nos conducirá a desarrollar competencia cultural, pues de eso se trata, de tener competencia y para ello es necesario además desarrollar habilidades de relación y comunicación y tener actitudes de consideración cultural, tales como el respeto, la confianza y la sensibilidad.

Uno de los mayores problemas en la asistencia sanitaria son las dificultades expresadas en términos de barreras lingüísticas, es decir, no hablar el mismo idioma. Pero éste no es el único problema, sino que "las diferencias culturales y la falta de competencia comunicativa de los profesionales, en términos de conocimientos de los aspectos de la comunicación no verbal característicos de cada cultura, tienen un importante peso en este sentido" (Oliver, 2002).

La herencia cultural afecta al pensamiento y comportamiento del lenguaje en el estilo y forma de comunicación, y estas características serán diferentes según la cultura. Pero además esas diferencias se pueden encontrar también en una misma cultura en función del sexo, puesto que mujeres y hombres son educados de diferente manera en casi todas las sociedades humanas (Oliver, 2002).

El algunas sociedades tradicionales existe una segregación sexual de los espacios, de modo que al hombre le corresponde el espacio público y a la mujer el privado. Esta situación influye en la comunicación de las mujeres, que pueden tener asumido el silencio fuera de los espacios privados, como las mujeres musulmanas (Oliver, 2002).

La comunicación intercultural no solo implica el acercamiento a otras culturas, sino examinar la cultura propia. "Si se parte del principio ético de que todas las culturas son dignas y merecedoras de respeto, estaremos en una posición de igualdad frente a la persona quien recibe lo cuidados" (González, 2011).

La persona que migra, bien sea la enfermera o las personas a las que atenderá, deben adaptarse al país de destino y a su contexto social, económico y cultural, pero no siempre éste es un paso fácil de asimilar y aceptar, puesto que las creencias culturales van con la persona y en algún momento, se producirá un encuentro cultural, que puede o no producir un choque.

Parece que "las enfermeras tienden a pensar que las diferencias en el modo de pensar o afrontar una situación de salud son fruto de creencias infundadas por parte de las personas de otras culturas, de falta de conocimiento o poca responsabilidad en su proceso de salud" (Roca, 2009). En muchas ocasiones, el discurso mediático proyecta una imagen negativa de la inmigración, asociándola con problemática social y elaborando la figura de un ser pobre a quien se debe compadecer.

No debemos caer en conductas paternalistas ni todo lo contrario, sino atender a la persona de otra cultura como lo haríamos con cualquier otra persona, respetando sus diferencias individuales y dando calidad a nuestro cuidado.

Todas estas situaciones y más, que se pueden dar en el contexto de un choque cultural o no, deben hacernos reflexionar y actuar con 
conocimiento de las leyes, como pueden ser: la ley de autonomía del paciente y de derechos y obligaciones en materia de información, y códigos éticos de la enfermería. De igual modo, es necesario garantizar que todas las personas tengan accesibilidad al sistema sanitario y eliminar las barreras que impiden una atención de sus necesidades.

Ante la diversidad cultural que impera en nuestra sociedad, ¿Qué medidas o herramientas podemos utilizar para mejorar la atención sanitaria? Por un lado, se puede recurrir a estrategias relacionadas con el marco sanitario, que incluirían: la incorporación de mediadores culturales para facilitar la accesibilidad y la equidad, elaboración de guías de salud en diversos idiomas, la creación de espacios de diálogo con asociaciones de inmigrantes y la formación de las enfermeras en interculturalidad.

El mediador cultural es una figura que empieza a tener su importancia y consideración en el sistema sociosanitario. Se trata de una "persona que actúa de puente de comunicación entre el profesional sanitario y el usuario y juega un papel activo y delicado al facilitar la relación terapéutica y neutralizar las diferencias culturales entre ambas partes, que no solo hablen idiomas diferentes, sino que tengas distintas percepciones de ver el mundo" (Delgado, 2011).

Algún autor opina que solicitar mediadores culturales implica delegar, pasividad y desprendimiento de responsabilidades; "distinto es el problema de la barrera lingüística para el que sí se puede necesitar pero es erróneo referirse a ello como la panacea universal a las dificultades derivadas de trabajar con personas de otras culturas" (Gentil, 2010).

Desde mi punto de vista, adquirir competencias en enfermería transcultural, implica mediar en situaciones culturales, implica co- nocimiento, sensibilidad y deseo de aprendizaje, pero no debemos olvidar que somos enfermeras, que nuestro base es ofrecer cuidados de calidad y no podemos otorgar un carácter heroico a nuestra asistencia, porque es difícil que sin tener una dedicación exclusiva al campo de la enfermería transcultural, se obtenga un conocimiento profundo de cada cultura e idioma. Es por ello, que el papel del mediador cultural es un recurso externo de utilidad, al igual que las guías de idiomas en salud y los espacios de encuentro cuando ha fallado ese primer contacto de relación entre enfermerausuario, y por ello creo que se debe fomentar su introducción, pero sin olvidar nuestra responsabilidad con las personas de otra cultura. Hablo de establecer un trabajo en red, de no tener miedo a trabajar en equipo multidisciplinar, de optimizar los recursos disponibles y ponerlos siempre en favor de las personas que atendemos.

No obstante, la principal medida es formarse, conocer, comprender y respetar las creencias de cada cultura y poner todo el trabajo y el esfuerzo por iniciar el camino de la formación intercultural para lograr ser enfermeras competentes en la sociedad actual.

La formación en competencia cultural forma parte de los estudios de grado y postgrado de Enfermería en países como Estados Unidos, Canadá, Reino Unido o Australia. En estos países, existen algunos hospitales en los que incluso se llegan a desarrollar entrenamientos específicos en competencia intercultural para profesionales y sólo se seleccionan aquellos que poseen formación específica en este campo (Plaza del Pino, 2009). Un estudio realizado por Plaza del Pino basado en indagar en el Libro Blanco del Título de Grado en Enfermería, pone de manifiesto que "existe un escaso interés desde la Enfermería en sus 
ámbitos académico, profesional y gestor en la formación de los nuevos profesionales para capacitarlos en el desarrollo de su trabajo en entornos multiculturales, aunque recientemente han aparecido cursos especializados, revistas y programas de doctorado específicos de cultura de los cuidados" (Plaza del Pino, 2009).

Revisando la Memoria de Verificación del Título de Grado en Enfermería de la Universidad de Oviedo se pueden ver competencias generales relacionadas con aspectos culturales, tales como: conocimiento de una lengua extranjera, trabajo en el contexto internacional, reconocimiento de la diversidad, conocimiento de otras culturas y costumbres, respeto a los derechos fundamentales de igualdad de oportunidades entre hombres y mujeres, solidaridad y cooperación al desarrollo, así como comprender el comportamiento de la persona en función del género, grupo o comunidad dentro del contexto social y cultural (Universidad de Oviedo, 2010). Sin embargo, si paralelamente se revisan las asignaturas y sus guías docentes se observa que no existe ningún contenido que pueda desarrollar esas competencias. Únicamente, en el plan de estudios de Enfermería desarrollado en Oviedo se incluye un epígrafe de habilidades de comunicación con pacientes de otra cultura enmarcado dentro de la asignatura de Habilidades Sociales. Por otro lado, en el plan de Estudios de Enfermería de Gijón (Centro Adscrito) incluye la asignatura optativa "Antropología de los cuidados" dedicada principalmente a conocimientos antropológicos que favorezcan el desarrollo profesional de la enfermera en una sociedad multicultural.

Esta realidad es inquietante ya que además de la necesidad de impulsar la formación en cuidados culturales por parte de las universidades, se necesita fomentar el interés de los estudiantes por este ámbito y eso no solo se consigue introduciendo una o dos asignaturas al respecto, sino promoviendo que estos conocimientos sean algo transversal en los estudios, implícito en cada asignatura. En mi opinión, el estudiante debe jugar un papel activo y participativo de su aprendizaje y resulta útil que en los nuevos modelos de enseñanza se estimule esa participación, involucrándolo en proyectos reales al margen de las clases magistrales, proyectos que las universidades deben ofertar, tales como programas de cooperación, voluntariado, integración o desarrollo de proyectos escolares, que impliquen al estudiante un contacto real y que favorezcan su interés y sensibilidad cultural.

\section{CONCLUSIONES}

Desde que la Enfermería Transcultural nació con Madeleine Leininger en los años 50, las enfermeras han aumentado su interés en considerar la cultura en la asistencia sanitaria, sobre todo en América y en la cultura anglosajona. Los conocimientos que se fueron incorporando en las enfermeras y en los sistemas políticos y sanitarios ejercieron su influencia en otros países de Europa, llegando poco a poco a España aunque no como un logro importante. No obstante, debido a la tendencia migratoria que ha aumentado considerablemente en nuestro país y se ha integrado también en el sector sanitario, las enfermeras y otros profesionales de salud empiezan a ser conscientes de la necesidad de adquirir competencia cultural que les facilite el establecimiento de las relaciones terapéuticas a través del entendimiento y la comprensión de su cultura. En general, las enfermeras españolas necesitan que las teorías y modelos de Enfermería con respecto a los cuidados culturales les sean prácticos y útiles para poder considerarlos, y todavía existen po- 
cos estudios y líneas de investigación abiertas con respecto a su utilidad práctica en nuestro entorno, por lo que sigue siendo una asignatura pendiente.

Esta área de conocimiento de la Enfermería Transcultural requiere compromiso de la enfermera, pues supone huir de los estereotipos y superar los prejuicios a fin de establecer una relación efectiva con el usuario, aceptando las diferencias culturales, viendo esto como una oportunidad de crecimiento y no como un problema.

El conocimiento de las otras culturas será un aspecto importante a considerar por las enfermeras, pero primará más la actitud sensible y de respeto hacia las personas de otra cultura, así como el estudio e importancia del contexto, como elemento clave de valoración enfermera, pues influye en la persona y en su afrontamiento ante las diversas situaciones de la vida.

Existe la necesidad de impulsar esta formación en las universidades, fomentando el interés de los estudiantes por éste ámbito, haciendo que sea una competencia transversal con un aprendizaje continuo, que fomente valores de solidaridad e innovación, a través de la participación activa en proyectos de colaboración, voluntariado, integración o proyectos escolares. No es suficiente que se integre una o dos asignaturas de estudio de los cuidados culturales sino se trata como un aspecto transversal de consideración en todos los ámbitos de la enfermería.

\section{BIBLIOGRAFÍA}

- Albornoz Mancera, D.M. (2009). La importancia de la transculturalidad en el conocimiento enfermero. Rev Paraninfo Digital, 3 (7), Disponible en: </para/n7/100d. php $>$. Consultado el 24 de Enero de 2014.

- Artigas Lelong, B., Vennasar Veny, M. (2009). La salud en el siglo XXI: el reto de los cuidados multiculturales.
Index Enferm, 18 (1), 42-46.

- Bonill de las Nieves, C., Celdrán Mañas, M. (2012). El cuidado y la cultura: Génesis, lazos y referentes teóricos en enfermería. Index Enferm, 21(3), 160-164. Consultado el 24 de Enero de 2014.

- Carro Furones, C., Camara Morales, E., Fomento Turné, G., López Gómez, M. (2009). Adaptación de enfermería al cuidado del paciente musulmán. Index Enferm, 3(7), Consultado el 24 de Enero de 2014.

- Chamizo Vega, C. (2005). Enfermería transcultural en España. Cultura de los Cuidados, 9(18), 19-23.

- Da Silva Vilelas, J.M., Dias Janeiro, S.I. (2012). Transculturalidade: $\mathrm{O}$ enfermeiro com competencia cultural. REME Rev min enferm, 16(1),120-127. Consultado el 15 de Febrero de 2014.

- Delgado Rodríguez, J., Expósito Barbuzano, A. (2011). ¿Qué puede aportar la mediación intercultural al ámbito sanitario? ENE Revista de Enfermería, 5(3), 59-65.

- Gentil García. I. (2010). Competencia cultural en Enfermería: población subsahariana. Cultura de los Cuidados, 14(27), 61-67.

- González Juárez, L., Noreña Peña, A.L. (2011). Comunicación intercultural como medio para favorecer el cuidado culturalmente aceptable. Rev ENEO-UNAM, 8(1), 55-60.

- Ibarra Mendoza, T. X., Siles González J. (2006). Competencia cultural, una forma humanizada de ofrecer cuidados de enfermería. Index Enferm, 55,44-48.

- Leno González, D. (2006). Buscando un modelo de cuidados de enfermería para un entorno multicultural. Gazeta de Antropología, 22 (32), 1-14. Disponible en: http://hdl.handle.net/10481/7118.

- Lillo Crespo, M., Casanoba Martínez, I. (2006). Fenómenos migratorios, competencia cultural y cuidados de salud. Cultura de los cuidados, 10(20), 87-91.

- Lillo Crespo, M., Vizcaya Moreno, F., Dominguez Santamaria, J.M., Galao Malo, R. (2004). Investigación cualitativa en enfermería y competencia cultural. Index Enferm, 44-45, 47-50.

- Lipson Juliene, G. (2000). Cultura y Cuidados de Enfermería. Index Enferm, 28-29, 19-25.

- Moreno Preciado, M. (2010). Nuevos enfoques en el cui- 
dado del “otro". Index Enferm, 19 (2-3), 167-171.

- McFarland, M. (2007). Teoría de la diversidad y de la universalidad de los cuidados culturales. En A. Marriner Tomey (Ed.), Modelos y teorías de Enfermería (pp.472497). Madrid: Elsevier.

- Ministerio de Empleo y Seguridad Social (2013). Extranjeros con certificado de registro y tarjeta de residencia en vigor [sede web]. Madrid: MESS Editor.

- Oliver Reche, Ma.I. (2002). Comunicación intercultural: comunicándonos con mujeres de otras culturas. Matronas Profesión, 9, 21-27.

- Padró Cabello, C. (2004). Aportaciones de la comunicación intercultural al ámbito de la salud. Educare 21, 7. Consultado el 4 de Marzo de 2014.

- Plaza del Pino, F. (2011). Ante la diversidad cultural construyamos una enfermería intercultural. Enfermería Comunitaria, 7 (1). Disponible en <http://www.index-f. com/comunitaria/v7n1/ec7102.php > Consultado el 31 de Enero de 2014 de http://www.index-f.com/comunitaria/v7n1/ec7102.php.

- Plaza del Pino, F.J., Soriano Ayala, E. (2009). Formación de los profesionales de enfermería: cuidar en la sociedad multicultural del siglo XXI. Index Enferm, 18(3),190-194.

- Roca, I., Capará, N., Vega Monteagudo, C. (2009). Vulnerabilidad social del colectivo inmigrante. Index Enferm, 3(7), 1-9.

- Universidad de Oviedo (2010). Memoria de Verificación del Título de Grado en Enfermería.. Disponible en: http:// www.unioviedo.es/euenferfisio/Memorias/enfer\%20Memoria\%201.pdf. Consultada el 20 de Marzo de 2014. 